Original Article

\title{
The when and who of social learning and conformist transmission ${ }^{2}$
}

\author{
Michael Muthukrishna ${ }^{\mathrm{a}, *}$, Thomas J.H. Morgan ${ }^{\mathrm{b}}$, Joseph Henrich ${ }^{\mathrm{a}, \mathrm{c}, \mathrm{d}}$ \\ a Department of Psychology, University of British Columbia, Vancouver, BC V6T 1Z4, Canada \\ b Department of Psychology, University of California, Berkeley, CA 94720, USA \\ c Vancouver School of Economics, University of British Columbia, Vancouver, BC V6T 1Z1, Canada \\ ${ }^{\mathrm{d}}$ Canadian Institute for Advanced Research
}

\section{A R T I C L E I N F O}

\section{Article history:}

Initial receipt 22 November 2014

Final revision received 21 May 2015

\section{Keywords:}

Cultural evolution

Social learning

Evolution

Conformist transmission

Culture

\begin{abstract}
A B S T R A C T
Formal evolutionary models predict when individuals rely on social learning over individual learning and the relative strength of their conformist social learning biases. Here we use both treatment effects and individual variation to test predictions about the impact of (1) the number of traits in an environment, (2) the adaptive or payoff relevance of those traits, (3) the fidelity of transmission, and (4) the size of groups. We find that both social learning and the strength of conformist transmission increase with the number of traits, the adaptive value of those traits, and the fidelity of transmission. The strength of conformist transmission increases with group size, but only when there were 2 traits in the environment. Using individual-level variation and recognizing that treatment effects predictably impact individuals differently, we show that IQ negatively predicts social learning, but has a U-shaped relationship to conformist transmission, suggesting strategic use of conformistbiased social learning among those with the highest IQ. Other plausible predictors, such as status, cultural background, and personality, were not predictive. Broadly, our results reveal that not only is the conformist transmission bias ubiquitous, but that past experiments, both human and non-human, have likely underestimated its prevalence and the prevalence of social learning by restricting designs to only 2 traits.
\end{abstract}

(c) 2016 Elsevier Inc. All rights reserved.

\section{Introduction}

Humans are a cultural species, heavily reliant on a rich repertoire of ideas, beliefs, values, and practices acquired from other members of their social groups. Evolutionary approaches to culture postulate that our species' social learning abilities - the psychological foundations that undergird these cultural repertoires - are genetically evolved cognitive adaptations for surviving in environments in which individually acquiring information is costly. Building on this, a large body of theoretical research has explored the conditions under which natural selection will favor various learning strategies (Boyd \& Richerson, 1985, 1988, 1996; Henrich \& Boyd, 1998, 2002; King \& Cowlishaw, 2007; Nakahashi, Wakano \& Henrich, 2012; Perreault, Moya, \& Boyd, 2012). This theoretical research provides clear predictions about when individuals, both human and non-human, should rely on their individual or asocial experience and when they should deploy one or more social learning strategies, such as conformist transmission (a tendency to disproportionately copy the majority or plurality). By contrast, relatively little empirical research has sought to directly test these models in the laboratory with human participants, though key exceptions with adult participants include McElreath et al. (2005), Efferson, Lalive, Richerson, McElreath, and Lubell (2008), and Morgan, Rendell, Ehn, Hoppitt, and Laland

\footnotetext{
Author acknowledges support from the Canadian Institute for Advanced Research. The authors acknowledge no potential conflicts of interest.

* Corresponding author. Tel.: + 16048283342 .

E-mail address: michael@psych.ubc.ca (M. Muthukrishna).
}

(2012) and with children include Wood, Kendal, and Flynn (2013), Haun, Rekers, and Tomasello (2012), Chudek, Brosseau-Liard, Birch, and Henrich (2013), and Morgan, Laland, and Harris (2014). Here, we aim to advance this research program empirically by testing some novel predictions and implications derived from existing theoretical work, as well as to replicate some prior results in new and more diverse populations. We test predictions regarding how (a) the number of cultural traits, (b) payoffs associated with different decisions, (c) fidelity of social transmission, and (d) group size influence the use of social over asocial learning, and the application of conformist biases within social learning. In addition, we consider the implications of existing models for predicting who might tend to use which strategies, and use individual differences in cognitive abilities, social status, and cultural background to account for individual level variation in learning strategies (for a similar effort in other transmission contexts see Flynn and Whiten (2012)). Our efforts extend prior research on conformist biased social learning, which revealed much individual variation, but did not attempt to account for it.

\subsection{Theoretical research}

Several evolutionary models (Boyd \& Richerson, 1985, 1988, 1996; Henrich \& Boyd, 1998) predict that reliance on social learning (over asocial learning) should increase with the cost or difficulty of asocial learning, the size of the majority, and the stability of the environment. These predictions make intuitive sense - individuals will prefer cheap, reliable, and accurate information; the reliability of social 
information increases with larger majorities and accuracy decreases with changes to the environment to which it pertains. Other models (King \& Cowlishaw, 2007) predict that reliance on social learning should increase with access to more demonstrators, which typically increases with group size: More demonstrators reduce sampling error.

Within the realm of social learning, evolutionary models reveal the social learning strategies (Laland, 2004; Rendell et al., 2011) and biases (Boyd \& Richerson, 1985) favored by different situations or circumstances. One such bias is conformist transmission. In a particular population, there may be many variants in behaviors, beliefs, or values, from herein referred to as traits. Conformist transmission (Boyd \& Richerson, 1985 ) represents a type of frequency dependent social learning strategy in which individuals are disproportionately inclined to copy the most common trait in their sample of the population (e.g. individuals have a $90 \%$ probability of copying a trait that $60 \%$ of people possess). Conformist transmission is particularly important, because it tends to homogenize behavior within groups, increasing between group variation relative to within group variation (Boyd \& Richerson, 1985; Henrich \& Boyd, 1998), strengthening the effect of intergroup competition on cultural variation (Chudek, Muthukrishna, \& Henrich, 2015; Henrich, 2012), and potentially hindering cumulative cultural evolution within a group (Eriksson, Enquist, \& Ghirlanda, 2007). Conformist transmission contrasts with unbiased transmission, whereby individuals copy a trait at the frequency found in the population (e.g. individuals have a $60 \%$ probability of copying a trait that $60 \%$ of people possess).

Several evolutionary models reveal the conditions when the conformist transmission bias is more adaptive than unbiased transmission. Typically, these models have analyzed only 2 traits. However, Nakahashi, Wakano, and Henrich (2012) have extended these models to $N$ traits. Their model predicts that the strength of the conformist bias will increase with the number of traits in the environment. To understand the logic, consider a world with only 2 traits-black and white shirts. The presence of black shirts at anything above $50 \%$ suggests that people are selecting black shirts above chance. However, in a world with four traits - black, white, green, and red shirts - black shirts need only be present above $25 \%$ to suggest selection above chance. Thus, if $51 \%$ of people were clothed in black shirts, you would be much more likely to also wear a black shirt if there were 4 shirt options than 2 and even more so if there were 10 options and so on. One important implication of this model is that all current models and experiments may have been underestimating the strength of the conformist bias, because there are often more than 2 traits in the real world. In addition to the number of traits, the model also predicts that the strength of the conformist bias will increase with errors in transmission and with strength of selection ${ }^{1}$ consistent with other 2 trait conformist bias models (Henrich \& Boyd, 2002). Other models (Perreault et al., 2012) predict that a stronger conformist bias will be more adaptive in larger groups, as information reliability increases, with an asymptotic relationship between group size and the strength of the conformist bias.

\subsection{Experimental research}

In contrast to the growing body of theory, there has been relatively little experimental research investigating conformist biases. The first experimental test of these theories explored the effects of task difficulty and environmental variability (McElreath et al., 2005). The results revealed both unbiased and conformist transmission, with increased conformist transmission as the environment fluctuated. However, the results were inconsistent between experiments and were ultimately difficult to interpret. A later experiment by Efferson et al. (2008) separated participants into asocial and social learners and looked for evidence of a conformist bias among the social learners. On average, participants exhibited a conformist bias, but there was also considerable

\footnotetext{
${ }^{1}$ We infer this last prediction based on migration less than $50 \%$ and weak selection (see Supplementary Materials, available on the journal's website at www.ehbonline.org).
}

variation within participants, including some non-conformists. Most recently, Morgan et al. (2012) systematically tested nine theoretically derived hypotheses, including hypotheses related to group size, majority size, confidence, asocial learning cost and difficulty, number of iterations, participant performance, and demonstrator performance. In all cases, the results supported evolutionary predictions and found evidence of a conformist bias. All three sets of experiments described above revealed heavy reliance on social learning and the presence of a conformist bias, but they also documented, but did not explain, substantial individual variation. In the present research, we test several evolutionary theories and address this gap.

\subsection{Present research}

In two experiments, we measure both the reliance on social learning and the strength of the conformist bias, testing several untested theoretical predictions. Based on the models, we predict that reliance on social over asocial learning will increase with: (a) transmission fidelity (Boyd \& Richerson, 1985, 1988, 1996; Henrich \& Boyd, 1998) and (b) group size (King \& Cowlishaw, 2007; Perreault et al., 2012). We predict that the strength of the conformist bias will increase with (a) number of traits (Nakahashi, Wakano, \& Henrich, 2012), (b) payoffs of the traits being copied (effectively the strength of selection; Nakahashi et al., 2012), and (c) errors in transmission (Henrich \& Boyd, 2002; Nakahashi et al., 2012). Note that as transmission fidelity increases (i.e. errors in transmission decrease), reliance on social learning is expected to increase, but the strength of the conformist bias is expected to decrease. The decrease in the strength of the conformist bias with increased transmission fidelity may be more intuitive if you consider that the conformist bias helps to correct for errors in transmission. As errors increase, it pays to put more weight on larger majorities since they're less likely to emerge by chance. In testing these predictions, we also tested the effect of majority or plurality size in a more ethnically diverse population than past conformist transmission experiments.

We also developed and tested hypotheses to account for individual differences in social learning and conformist transmission. No work has yet shown what accounts for these differences, nor applied theoretical insights to understand the variation. Applying existing theory to individual variation, we explored three individual difference measures:

a) Cognitive abilities: Individuals with better cognitive abilities ought to possess better private information, resulting in less individual uncertainty, which should result in reduced reliance on social learning and conformist transmission. Alternatively, those with better cognitive abilities may select the more adaptive strategy (i.e. copying when uncertain) - that is, cognitive abilities may in part be about selecting the best learning strategy overall.

b) Status: Individuals who perceive themselves as higher in prestige status may reduce their reliance on learning from others who they perceive as less prestigious. Dominance status will bear no relationship to learning strategies once we control for prestige status and cognitive abilities.

c) Cultural Background: Populations may differ in their tendency toward social learning and conformist transmission (Bond \& Smith, 1996; Cialdini, Wosinska, Barrett, Butner, \& GornikDurose, 1999; Mesoudi, Chang, Murray, \& Lu, 2015). Cultural psychologists have argued that East Asians in particular are more likely to conform than Westerners. This may result in population-level differences in social learning and conformist transmission.

Besides these theoretically motivated variables, we also examined individual differences in (1) reflective thinking styles (intuitive vs reflective), (2) rule following, (3) personality, and (4) a variety of demographic variables. 


\section{Methods}

We ran both our experiments on the same participants, but randomized the order of measures and experiments between groups. We report our participant demographics, general design, and specific procedures for each experiment.

\subsection{Participants}

We recruited 101 participants from the University of British Columbia's Economics Participant Pool, which is open to the public, but primarily consists of undergraduate students. Of these 101 participants, 27 participants failed at least one of our two vigilance check questions, leaving us with 74 usable participants (39 Female; Mean Age $=21.73$, $\mathrm{SD}=5.55$ ). Including all participants is arguably defensible for our contextual variable analyses, because participants were incentivized for performance. Their inclusion generally strengthens our overall findings. However, since these participants were not incentivized for completing the individual-difference measures and failed vigilance checks within them, we conservatively exclude them from the main analysis, but report all analyses with their inclusion in Supplementary Materials (available on the journal's website at www.ehbonline.org).

\subsection{General design}

We ran two experiments on all participants. In Experiment 1, we examined the effects of the number of traits. In Experiment 2, we tested the effects of payoffs and transmission fidelity. In both studies, we also explored group size (from 5 to 11 participants) and the proportion of people who selected each trait. In our experiment, traits are the lines of different length that participants selected between; we will refer to them as options from herein. As noted, we also measured several individual-level factors, detailed in Background Measures. Participants were paid a show-up fee of $\$ 10$ and could win an additional $\$ 20$ based on performance in the two experiments. Fig. 1 illustrates the general design of the experiment.

\subsection{Experiment 1: number of options}

In Experiment 1, participants had to compare between 2 and 6 lines to identify the longest line. This was repeated 10 times. The lines appeared for $3 \mathrm{~s}$ and then participants made their first 'asocial' decision. The software then displayed the decisions made by other participants one after another. The participants were shown panels corresponding to the different lines and each decision made by another participant was indicated by the corresponding panel flashing (red then gray). After receiving this social information, participants answered the question again. Keep in mind that there was no deception in this experiment, so this was real social information.

Each trial was worth up to $\$ 1$. The payoff associated with each line was proportional to the length of the selected line relative to all other lines, with the longest line worth $\$ 1$, the shortest line worth nothing, and lines of intermediate length worth a value less than $\$ 1$ based on the function graphed in Fig. S1 (see Supplementary Materials for details, available on the journal's website at www.ehbonline.org). With 10 trials each worth a maximum of $\$ 1$, participants could earn $\$ 10$ in this phase of the session. We informed participants at the beginning of the experiment that their payment depended only on their second response to each set of lines.

\subsection{Experiment 2: transmission fidelity and payoffs}

In Experiment 2, we restricted the number of lines to 2 and varied the transmission fidelity and payoffs. The task involved comparing 30 pairs of lines to identify the longest line, with participants first giving an asocial response and then receiving social information and information about transmission fidelity before getting a chance to answer again. In other respects, participants went through the same process as in Experiment 1.

To explore the impact of transmission fidelity, we varied errors in transmission by replacing some of the social information with random computer generated answers. We informed participants of the probability of replacing real social information, which ranged from $0 \%$ (only true social information) to $40 \%$ (i.e. $60 \%$ social information, $40 \%$ random). See Supplementary Materials, available on the journal's

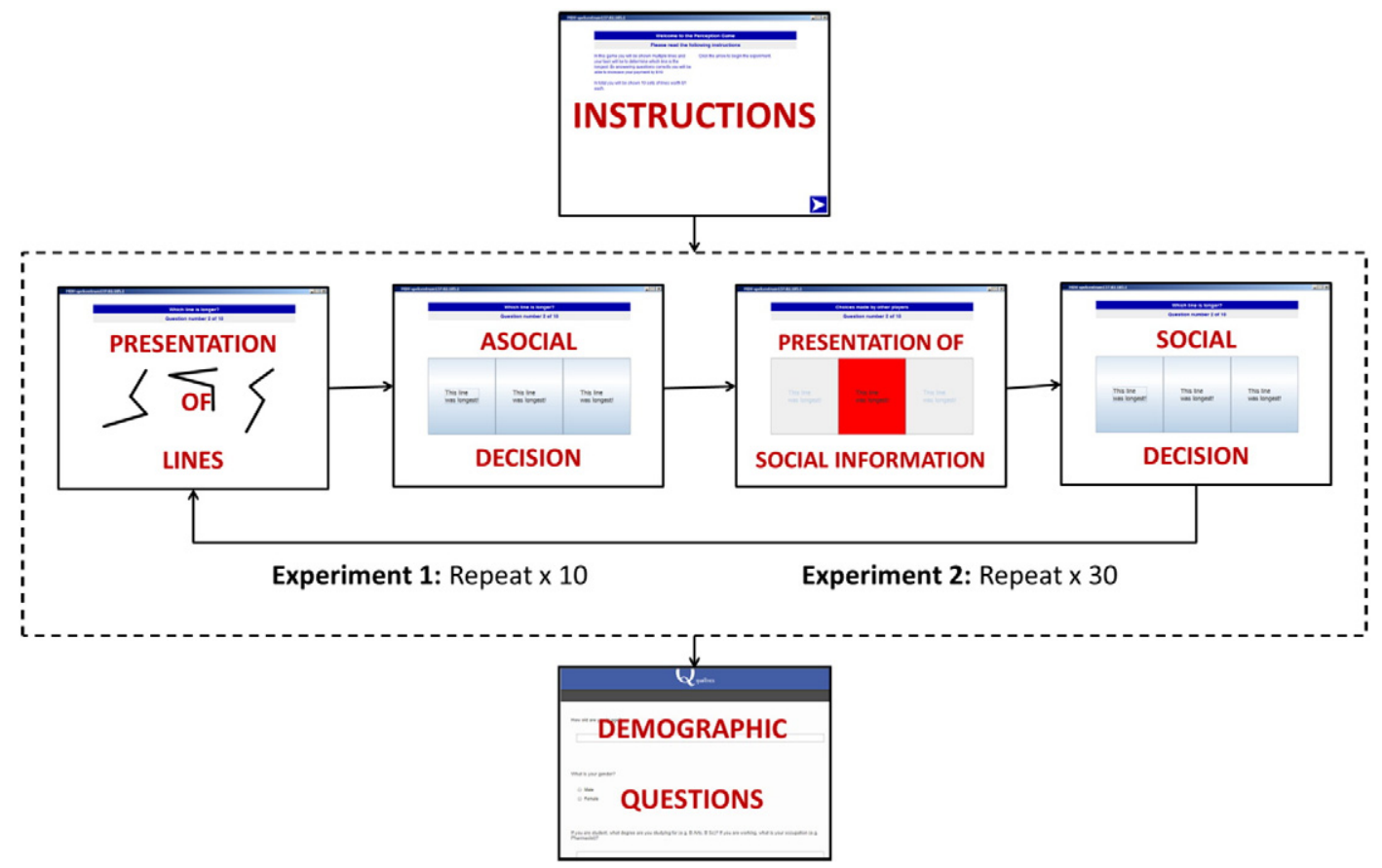

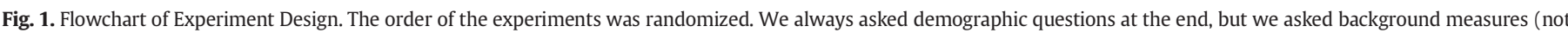
shown) before or after all experiments (also randomized). 
website at www.ehbonline.org for a screenshot and details. After receiving this noisy social information, participants made their final decision.

To explore the impact of payoffs, we made the value of each trial between $\$ 0$ and $\$ 2$, with the ability to earn up to $\$ 10$ over 30 trials. The software clearly indicated the amount of money each question was worth before and throughout each trial.

We administered background measures either before or after the two experiments (randomly assigned with no significant difference between behavior or measures), but demographic questions (age, sex, time spent in Canada (some participants are immigrants), strategies used while playing the game, etc.) were always asked at the end.

\subsection{Background measures}

Our three key individual-difference predictors were:

- IQ: We measured IQ using Raven's Advanced Progressive Matrices (Raven \& Court, 1998).

- Prestige and Dominance: We measured self-reported prestige using the Prestige and Dominance scale (Cheng, Tracy, \& Henrich, 2010).

- Cultural Background: We asked for participant ethnicity, if they had lived their entire lives in Canada, how well they speak their native language, how much they identify with Canada (Inclusion of Other in the Self Scale; Aron, Aron, \& Smollan, 1992), and their degree of acculturation (Vancouver Index of Acculturation; Ryder, Alden, \& Paulhus, 2000).

To pre-emptively counter other potential explanations for variation in social learning and conformist transmission, we also measured:

- Reflective vs Intuitive Thinking Styles: We measured reflective vs intuitive thinking styles using the Cognitive Reflection Test (CRT; Frederick, 2005). We included the CRT since it is plausible that copying or not copying others may be an intuitive decision. In this case, intuitive or reflective thinking styles will predict social learning and conformist transmission.

- Rule Following: We measured the tendency to follow rules using the Rule Following Task (RFT; Kimbrough \& Vostroknutov, 2013). We included the RFT since it is plausible that copying or not copying simply represents the rule in our experimental setting, in which case the tendency to follow rules will predict social learning and conformist transmission.

Finally, we included age, sex, and the Big 5 Personality Inventory, which are often a source of individual-differences. Further details can be found in Supplementary Materials (available on the journal's website at www.ehbonline.org).

\section{Analysis}

Our first theoretical question concerns how our contextual variables influenced social learning and conformist transmission. In our analysis of social learning, we looked at the proportion of times participants changed their decision after viewing social information for each level of our predictor variables. We graphed these relationships and described them with a best-fitting function, and then predicted this binary decision (changed vs did not change) using our predictor variables. This analysis allowed us to look at how our manipulated predictors affected the use of social information, but we could not use the proportion of participants as a predictor, since those in the majority or plurality would themselves be less likely to change their decision.

To address the question of how majority size affected social learning with 2 traits, we followed Morgan et al. (2012): Participants are considered to have used social information if (a) their decision after viewing social information differed from their asocial decision and (b) the majority of other participants disagreed with the participant's original decision. In Experiment 1, there were pluralities rather than majorities (multiple options), and there was more information (e.g. relative proportions), which participants may have incorporated in addition to just the overall plurality. Here, we analyzed the data with all responses (not just where the plurality disagreed with the participant), but focused on the cases where participants changed their decision. In each case where a decision was changed, we looked at the frequency of each option; the frequency of the options the participants ultimately selected and the frequency of the options the participants did not ultimately select.

Finally, to determine the strength of any conformist bias, we ran an analysis where we calculated a single best-fit conformist transmission parameter $(\alpha)$ by aggregating the data across all individuals for each level of our key predictors - number of options, transmission fidelity, and payoff value - except group size, where we did not have enough participants in each level. To accomplish this, we used a Signal Detection Theory (SDT) perspective, considering the four possible decision scenarios for a particular option and frequency. Note that this is for each particular option. To illustrate, we use Line 2 (of between 2 and 6 lines) as the particular option:

SDT 1 . Choosing the option both asocially (before seeing social information) and socially (after seeing social information). E.g. Line 2 is selected before seeing social information and Line 2 is selected again after seeing social information.

SDT 2. Choosing the option asocially, but choosing a different option socially. E.g. Line 2 is selected before seeing social information, but a different line (not Line 2 ) is selected after seeing social information.

SDT 3. Choosing a different option asocially, but choosing the option socially. E.g. a different line (not Line 2 ) is selected before seeing social information, but Line 2 is selected after seeing social information.

SDT 4. Choosing a different option asocially and socially. E.g. a different line (not Line 2 ) is selected before seeing social information and a different line (not Line 2 ) is selected after seeing social information.

In SDT 1, we have no way of assessing if a decision was based on the social information or asocial prior. In contrast, in the other three cases, we know that the proportion was insufficient to retain the decision (SDT 2), the proportion was sufficient to make them choose the option (SDT 3), or the proportion was insufficient to make them choose the option (SDT 4).

We used a logistic function to fit a sigmoid to these latter three cases (SDT 2-4), similar to earlier theoretical work in social learning (McElreath et al., 2008; Szabó \& Tőke, 1998; Traulsen, Pacheco, \& Nowak, 2007):

$$
p_{i}=\frac{1}{1+e^{-\alpha\left(p_{t}-c\right)}}
$$

Where $p_{i}$ is the probability of choosing option $t$ and $p_{t}$ is the frequency of option $t$. The $\alpha$ parameter of the sigmoid is a measure of the strength of the conformist bias. If $\alpha<0$, this indicates anti-conformity and if $\alpha \approx 0$, we assume that decisions are being made independent of social decisions, i.e. no social learning. In contrast, $\alpha<5$ suggests some social learning, but not conformist transmission. Finally, $\alpha \geq 5$ is evidence of conformist transmission, with higher values indicating a stronger conformist transmission bias. The $c$ parameter tells us the inflection point, i.e. when individuals are $50 \%$ likely to choose the option and suggests a conformist bias when $c<0.5$. These four categories match four types of formally defined frequency-dependent social learning strategies, which we discuss in Supplementary Materials (available on the journal's website at www.ehbonline.org).

Nakahashi et al. (2012) predict that $c$ should be inversely related to the number of options $(N)$, i.e. $c=1 / N$ - this is the frequency at which the trait would be present at chance levels. We used a nonlinear least- 
squares (NLS) estimate to fit $\alpha$ and $c$ in Experiment 1 with multiple options, measuring the strength of the conformist bias and testing the theoretical predictions of Nakahashi et al. (2012). In Experiment 2, with only 2 options, we set $c=0.5$, the expected inflection point $(c=1 / 2)$ to fit the strength of the conformist bias $(\alpha)$. In Fig. 2, we plot the sigmoid based on this function for different values of $\alpha$ and $c$.

Our second theoretical question was what individual factors predicted the strength of conformist transmission. To answer this second question, we fit the strength of conformist transmission to all responses for each individual separately. We then regressed these individual-level $\alpha$ values on our individual-level predictors.

\section{Results}

We report the results for contextual predictors and then individual predictors, analyzing Experiment 1 and 2 separately. We analyze the effect of each predictor on social learning and then the strength of the conformist bias.

\subsection{Number of options (Experiment 1)}

Recall that in Experiment 1 participants had to select the longest line from between two and six options. We begin by analyzing the effect of the number of options on people's reliance on social learning over asocial learning.

\subsubsection{Social learning}

Fig. 3 shows a non-linear relationship between the number of options and the percentage of decisions that changed after seeing social information. With only 2 options, a little over $10 \%$ of people changed their decision after viewing social information, but this number rises to over $25 \%$ with 4 options and to almost $30 \%$ with 6 .

Next, we look at how the frequency of each option in the social information predicted changing to that option. To do this, we use a binary logistic model to regress participant's decisions on the proportion of participants who selected an option (Proportion), the number of options (Options), and number of participants in the group (Participants), thereby testing several theoretical predictions (Boyd \& Richerson, 1985, 1988, 1996; Henrich \& Boyd, 1998; King \& Cowlishaw, 2007). Each participant made multiple decisions. We control for common variance created by multiple observations from the same person with random effects for each individual. We remove age and gender from the analysis; neither was significantly predictive and made very little difference to the results (see Supplementary Materials for full models, available on the journal's website at www.ehbonline.org). Nakahashi et al. (2012) made no specific predictions about the functional form of the

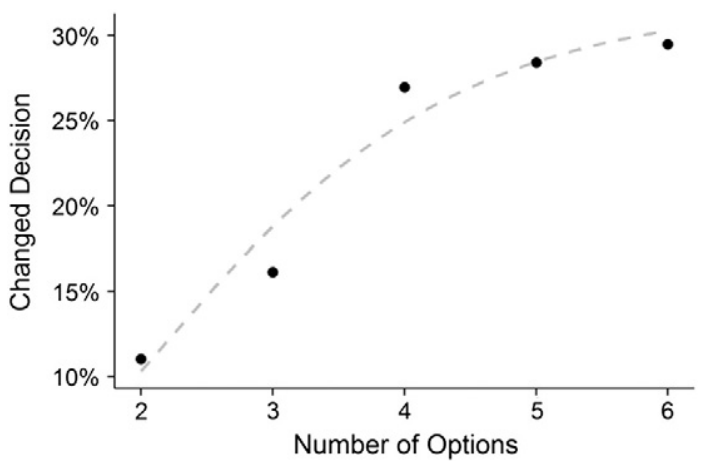

Fig. 3. Percentage of decisions that were changed after seeing social information for different number of options. Although there are too few points to be certain about the function that best fits these data, we used a non-linear least squares method to fit to the reciprocal of the number of traits $\left(y=-0.60^{1} / x n+0.40\right)$, plotted with a gray dashed line. Our choice of fitting the reciprocal of the number of traits is based on the logic underlying the Nakahashi et al. (2012) model i.e. the probability of selecting the trait at chance is $1 / n$.

relationship between the rate of social learning and number of traits. But, guided by their predictions for the conformist bias and predictions made by other models for the effect of the cost of asocial learning (which should increase with more traits), we test a model with the number of options (Model 1) and a model with the reciprocal of the number of options $(1 /(N-1)$; Model 2$)$. We report these in Table 1.

Table 1 reveals that participants are much more likely to change their decision overall if there are more options -1.68 times as likely for every additional option. Participants are also more likely to change their decision as the proportion of others who select the option increases -3.6 times as likely for every additional $10 \%$ of participants. Our results indicate that the number of participants in the group (5-11) did not affect the likelihood of changing the decision. Based on the AIC values (Table 1) the fit of the number of options model and that of the reciprocal of options model were almost identical.

\subsubsection{Conformist bias}

To examine the influence of multiple options on the strength of the conformist bias in social learning, we fit the logistic function described in the Analysis section to the frequencies participants saw and their decisions for each number of options. We did this by combining all participants for each level of options $-2,3,4,5$, and 6 . Thus, for each number of options, we calculate the strength of conformist bias $(\alpha)$ and the inflection point $(c)$, i.e. what percentage of demonstrators need to have selected an option for the participant to copy that option with a $50 \%$ likelihood.
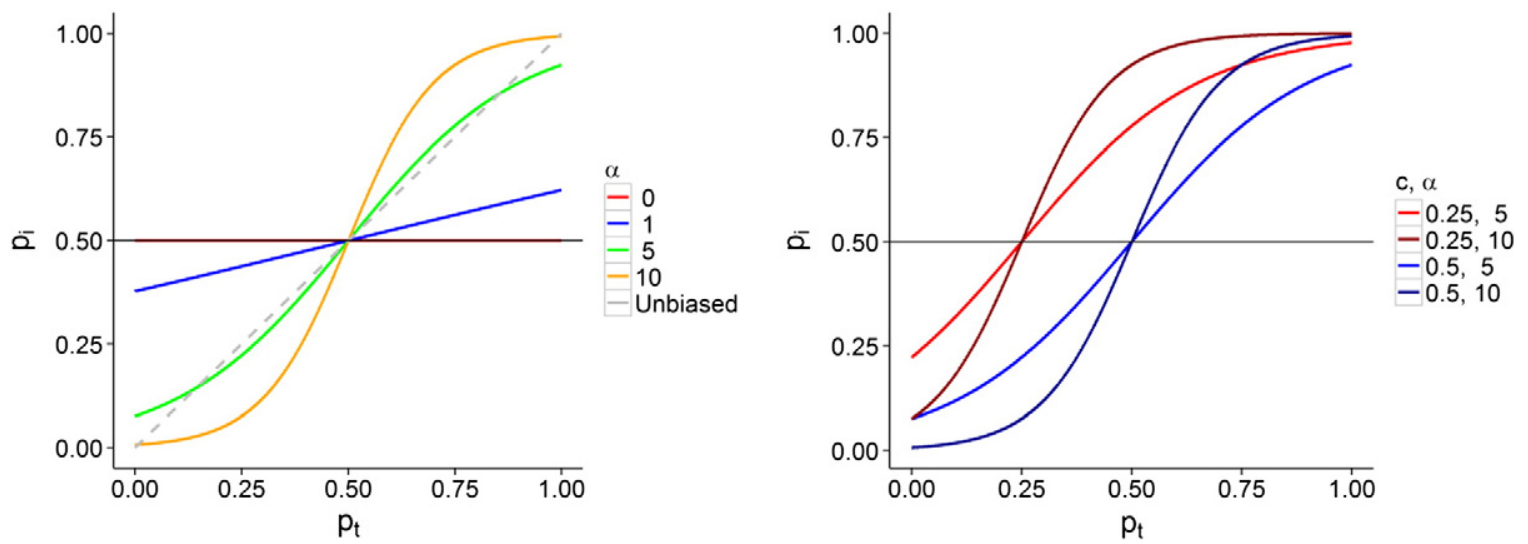

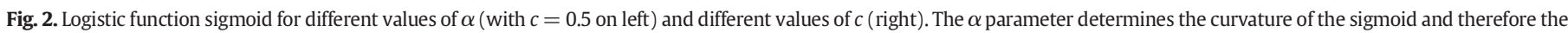
strength of the conformist transmission bias. The $c$ parameter determines the inflection point. 
Table 1

Binary logistic multilevel model of decision to switch regressed on the proportion of participants in the option (in 10\% increments for easier interpretation), the reciprocal and number of options (separate models), and the number of participants in the group.

\begin{tabular}{llll}
\hline & Model 1 & Model 2 \\
\cline { 2 - 2 } & Number of Options & & Reciprocal of Options \\
\hline (Intercept) & $<0.01^{* * * *}[0.00,0.01]$ & & $0.05^{* *}[0.01,0.42]$ \\
Proportion $10 \%$ & $3.62^{* * *}[2.82,4.87]$ & & $3.56^{* * *}[2.79,4.78]$ \\
Options & $1.68^{* *}[1.23,2.35]$ & & $0.04^{* *}[0.01,0.32]$ \\
Participants & $0.96[0.76,1.21]$ & $0.92[0.73,1.16]$ \\
AIC & 171.67 & 173.13 \\
Obs. & 332 & 332 \\
Groups & 64 & 64 \\
\hline
\end{tabular}

All coefficients are odds ratios. We control for common variance created by multiple observations from the same person with random effects for each individual.

${ }^{*} \mathrm{p}<.05$.

** $\mathrm{p}<.01$.

*** $\mathrm{p}<.001$

Fig. 4a reveals that with each additional option, the strength of the conformist bias increases, but consistent with Nakahashi et al. (2012), the size of each increase decreases. Fig. $4 \mathrm{~b}$ reveals that the inflection point decreases reciprocally with increasing options, as predicted by the model of Nakahashi et al. (2012), though the actual value is higher than theoretical predictions (shown as a solid line to distinguish it from dashed lines fitted to the data). The difference between the experimental measurements and theoretical prediction may be an indication of the size of participants' asocial prior, which the model of Nakahashi et al. does not address - they model a situation where individuals can only access either asocial or social information (but not both). The pattern in Fig. $4 \mathrm{~b}$ is what one would expect if individuals can combine asocial and social learning, as is the case in our experiments.

Fig. $4 \mathrm{~b}$ reveals the point at which individuals will select an option $50 \%$ of the time (c). With only 2 options, individuals select an option $50 \%$ of the time if $75 \%$ of others select it. With 4 options, individuals select an option $50 \%$ of the time if $50 \%$ of others select it. And with 6 options, individuals select an option $50 \%$ of the time if just $35 \%$ of others select it. Fig. 4a reveals a measure of the gradient of the sigmoid $(\alpha)$. To get a sense for what these two parameters are telling us, consider what happens when someone sees $80 \%$ of other people select an option. If there are 2 options ( $\alpha=7$ and $c=.75$ ), the person has a 59\% probability of changing their decision, but if there are 6 options $(\alpha=17$ and $c=$ 0.35 ), the person has a $99.95 \%$ probability of changing their decision. Together, these results reveal that as the number of traits in an environment increases, both social learning and the strength of the conformist bias increase, but at a diminishing rate.

\subsection{Transmission fidelity and payoffs (Experiment 2)}

Experiment 2 varied errors in the transmission channel and payoffs. To remain consistent with most existing theoretical models and with prior experimental research, we restricted choices to 2 options (instead of the 2 to 6 options in Experiment 1). As for Experiment 1, we first examine how these 2 factors influence social learning, and then look at their effect on the strength of the conformist bias.

\subsubsection{Social learning}

Reliance on social information increased with higher fidelity transmission. Fig. 5a suggests a linear relationship between transmission fidelity and the percentage of decisions that changed after seeing social information. At $100 \%$ transmission fidelity, about $16 \%$ of people changed their decision after viewing social information, but this number drops to $11 \%$ at $60 \%$ fidelity. Though this increase with fidelity is consistent with theoretical expectations, the differences in social learning were small; participants were not particularly responsive to our rather explicit manipulation of transmission fidelity.

Reliance on social information increased between having no payoff and some payoff, but did not increase with higher payoffs. Fig. 5b shows that the percentage of decisions that changed after seeing social information increased by about 3\% in moving from a zero payoff to 10 cents, but then remained consistent between $13 \%$ and $15 \%$ up to payoffs of $\$ 2$. The difference between zero and even a small payoff is consistent with prior experimental work on the Zero Price Effect (Shampanier, Mazar, \& Ariely, 2007). One possible explanation for the lack of effect of increasing payoffs is that our experiment did not have the range or sensitivity to capture the effect of payoffs. For the transmission rates used in our experiment, Nakahashi et al. (2012) predict small and diminishing returns for low payoffs (weak selection in the model).

As in Experiment 1, we use a binary logistic multilevel model to regress participant decision on the size of the majority, transmission fidelity, question payoff, and number of participants in the group. We control for common variance created by multiple observations from the same person with random effects for each individual. We removed age and gender from the analysis; neither was significantly predictive and made very little difference to the results (see Supplementary Materials for full models, available on the journal's website at www.ehbonline. org). We consider majority percentage and transmission rate in $10 \%$ intervals and payoffs in 10-cent intervals for more intuitively interpretable coefficients (Model 1). We also ran a second model with payoffs as a binary variable with no payoffs vs non-zero payoffs (Model 2).

Table 2 reveals a large effect of majority percentage, such that every $10 \%$ increase is associated with participants being 3.5 times more likely (a)

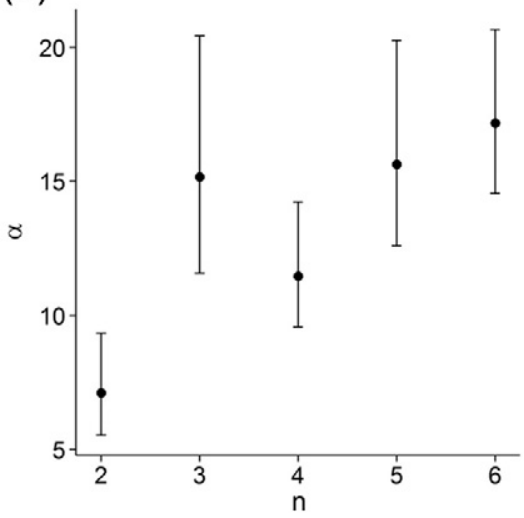

(b)

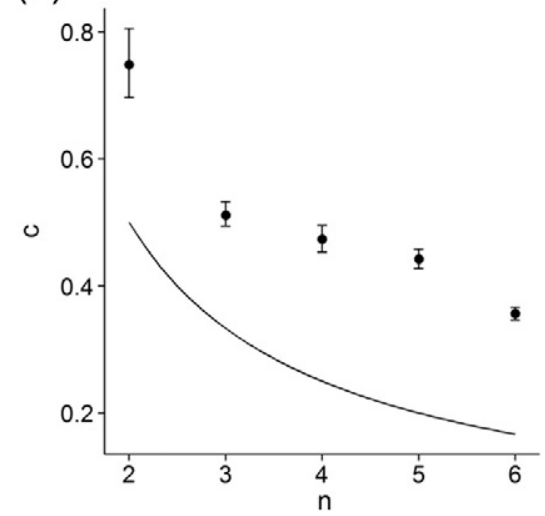

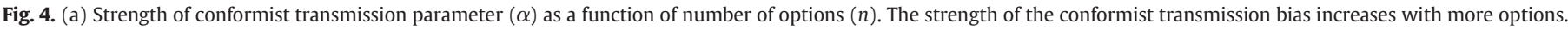

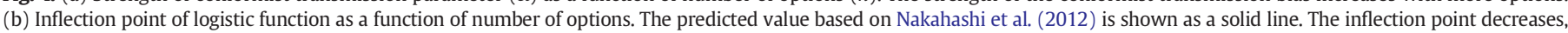
but remains higher than the predicted value, indicating an asocial prior. 
(a)

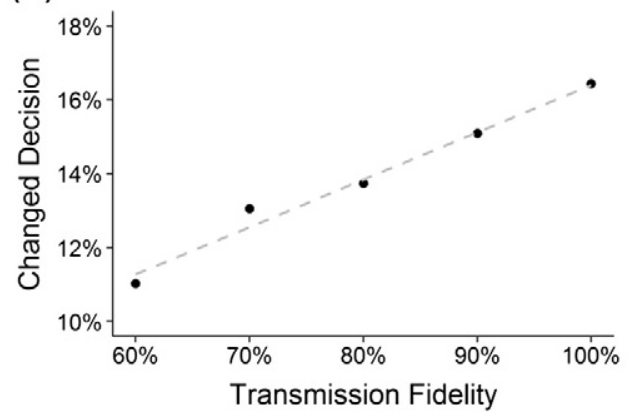

(b)

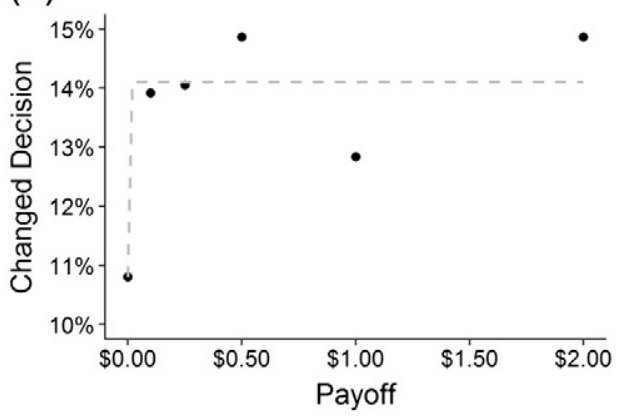

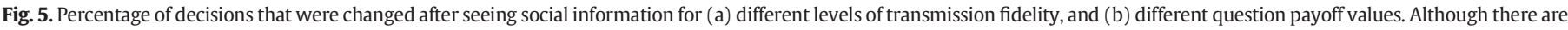

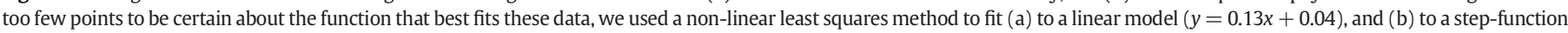
$(y=0.14$ if $x>0 ; y=0.11$ if $x=0)$. Fit functions are plotted with a gray dashed line.

to change to the majority. We also find a large positive effect of transmission fidelity, with every additional $10 \%$ increase in fidelity associated with participants 1.3 times as likely to change to the majority. Consistent with Fig. 5b, we see no linear effect of payoff, but a significant difference between zero payoff and non-zero payoffs (participants are 2.6 times as likely to switch to the majority with some payoff). Finally, every additional participant in the group results in participants 1.28 times as likely to switch to the majority. Except for payoffs, these results are consistent with our theoretical predictions (Boyd \& Richerson, 1985, 1988, 1996; Henrich \& Boyd, 1998; King \& Cowlishaw, 2007).

\subsubsection{Conformist bias}

To analyze the effect of the number of options on the strength of the conformist bias in Experiment 1, we fit the logistic function described in the Analysis section for 2 options, 3 options, and so on. Here, in Experiment 2 , we perform the same analysis for each level of transmission fidelity (60\%, 70\%, 80\%, etc.) and then each level of payoffs (0c, $10 c, 25 c$, etc.).

Transmission fidelity significantly increases the strength of the conformist bias between $60 \%$ and $70 \%$ fidelity, but there is no clear difference above $70 \%$ (see Fig. 6a). Recall that in contrast, social learning increases linearly with transmission fidelity. The difference in the strength of the conformist bias between $60 \%$ and $70 \%$ fidelity is large. An individual who sees $80 \%$ of others select an option will be $85 \%$ likely to copy that option if transmission fidelity is $60 \%$, but will be $95 \%$ likely to copy the option if transmission fidelity is $70 \%$.

Higher payoffs predict a stronger conformist bias (although the large confidence intervals make it difficult to determine if this trend is more than chance; see Fig. $6 \mathrm{~b}$ ). The very large confidence interval on $\$ 1$ and $\$ 2$ may be due to fewer cases for these values. To compensate for this, we averaged the $\$ 1$ and $\$ 2$ cases in Fig. 6 c. These results suggest that higher payoffs lead to a stronger conformist transmission bias, with

Table 2

Binary logistic multilevel model of decision to switch to majority on majority size, transmission fidelity, payoff, and number of participants in the group.

\begin{tabular}{llll}
\hline & \multicolumn{2}{l}{ Model 1} & Model 2 \\
\cline { 2 - 2 } & Linear Payoff 10c & & Binary Payoff \\
\hline (Intercept) & $1.22[0.81,1.84]$ & & $0.49[0.16,1.21]$ \\
Majority 10\% & $3.50^{* * *}[2.84,4.32]$ & & $3.60^{* * * *}[3.06,5.01]$ \\
Transmission 10\% & $1.29^{* * *}[1.12,1.49]$ & & $1.31^{* * * *}[1.13,1.55]$ \\
Payoff & $1.01[0.97,1.06]$ & & $2.62^{*}[1.16,7.79]$ \\
Participants & $1.28^{* *}[1.07,1.53]$ & & $1.28^{*}[1.09,1.59]$ \\
AIC & 761.47 & 750.10 \\
Obs. & 818 & 818 \\
Groups & 74 & 74 \\
\hline
\end{tabular}

All coefficients are odds ratios. We control for common variance created by multiple observations from the same person with random effects for each individual.

* $\mathrm{p}<.05$.

** $\mathrm{p}<.01$.

*** $\mathrm{p}<.001$ diminishing returns. Recall that we saw no trend in social learning, except between no payoff and some payoff. Thus payoffs have little effect on social learning, but do have an effect on the conformist social learning bias. Overall, these results only partially support the theoretical predictions. We will return to this in the Discussion.

\subsection{Individual variation in social learning strategies}

Consistent with past empirical research (Efferson et al., 2008; McElreath et al., 2005; Morgan et al., 2012), we found evidence of substantial individual variation in social learning and social learning strategies. We used the same analytic approach as in the previous sections analyzing social learning and then conformist transmission. To measure reliance on social information, we calculated the percentage of decisions that each participant changed after seeing social information. To measure the strength of the conformist bias $\left(\alpha_{i}\right)$, we fit a logistic curve based on the frequency of options they saw. We then regressed the social learning measure and the conformist bias measure on our theoretically motivated predictors (IQ prestige, and culture), as well as several other measures that have been used in the literature, including reflective thinking styles, rule following, personality, and a variety of demographic variables.

\subsubsection{Social learning}

In both experiments, IQ was significantly predictive of lower reliance on social information (see Table 3). Every standard deviation increase in IQ resulted in a 4\% reduction in social learning in Experiment 1 and a 2\% reduction in social learning in Experiment 2. This effect is small, but reliable.

With the exception of IQ no other predictors were reliably predictive. Neither prestige nor cultural background was sizably or significantly predictive. Nor were other plausible predictors such as reflective thinking styles, rule following tendencies, personality, dominance, and a variety of other demographic variables. Since our participants included partially acculturated individuals, we attempted to predict social learning using the interaction of cultural background and measures of acculturation and cultural identification. These were also not sizably or significantly predictive. However, all of our predictors together account for only about $9 \%$ of the variance in social learning. We briefly return to our null results with regard to cultural differences and prestige in the Discussion.

\subsubsection{Conformist bias}

To assess the variation in the strength of conformist biases in social learning, we fit a logistic curve to all participant responses in Experiment 1 and 2 separately, assuming an inflection point of $1 / N$ in order to fit the model. For all models, we again used a SDT approach, focusing on the 3 cases of interest and used the NLS method to estimate parameters. 
(a)

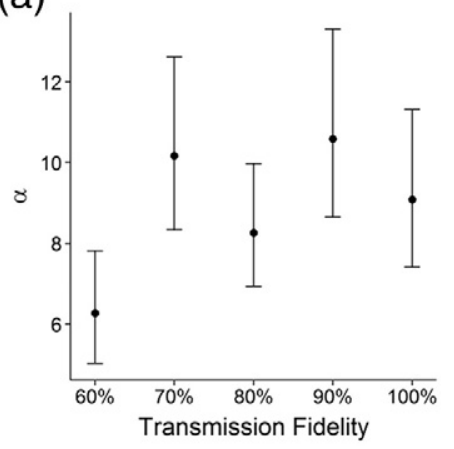

(b)

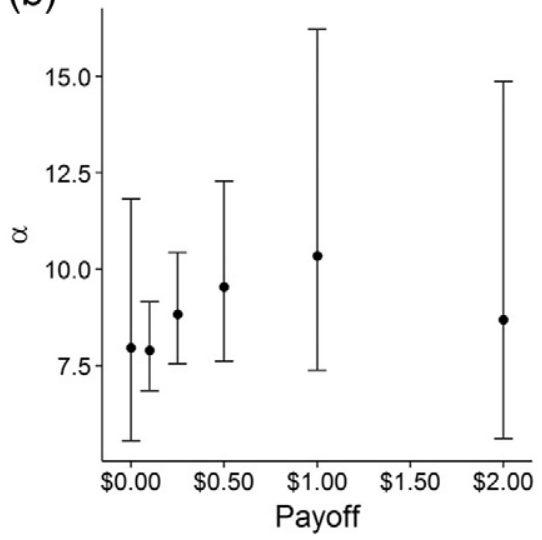

(c)

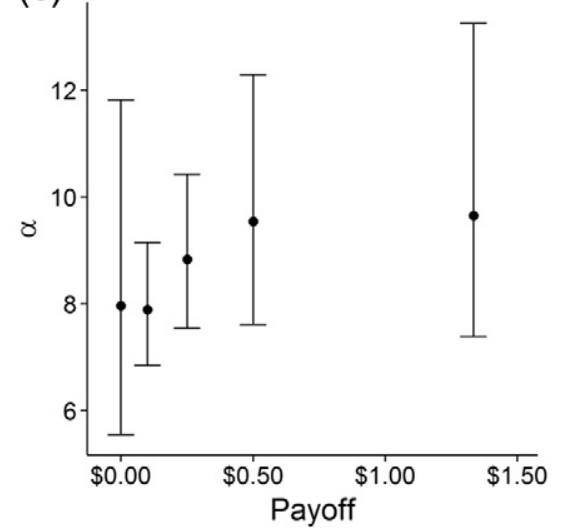

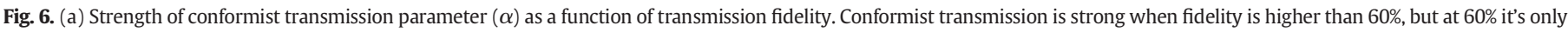

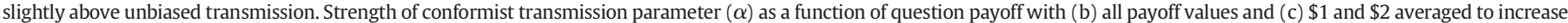
sample size for the highest value. The strength of the conformist transmission bias increases with diminishing returns as the payoffs increase.

Fig. 7 shows the distribution of $\alpha_{i}$ values in both experiments, with the vertical line marking unbiased, as opposed to conformist, transmission. In Experiment 1, only 3\% of people showed unbiased social learning (or weaker). The remaining $97 \%$ of participants showed a conformist transmission bias to varying degrees, with the modal value a bit above 10. We found no evidence of anti-conformity. In Experiment $2,15 \%$ of participants showed unbiased social learning (or weaker)

Table 3

OLS regression model percentage of decisions that were changed after viewing social information regressed on theoretical predictors as well as age and gender.

\begin{tabular}{lll}
\hline & Experiment 1 & Experiment 2 \\
\hline (Intercept) & $0.22(0.15,0.30)^{* * *}$ & $0.20(0.02,0.39)^{*}$ \\
zIQ & $-0.04(-0.08,-0.00)^{*}$ & $-0.02(-0.05,-0.00)^{*}$ \\
zPrestige & $-0.01(-0.05,0.02)$ & $-0.01(-0.05,0.02)$ \\
East Asian & $0.00(-0.09,0.09)$ & $-0.01(-0.06,0.04)$ \\
Other Ethnicity & $-0.02(-0.12,0.08)$ & $0.03(-0.03,0.10)$ \\
zAge & $-0.00(-0.04,0.04)$ & $-0.00(-0.03,0.02)$ \\
Male & $0.01(-0.06,0.09)$ & $-0.00(-0.05,0.04)$ \\
$\mathrm{R}^{2}$ & 0.09 & 0.09 \\
$\mathrm{~N}$ & 74 & 74 \\
\hline
\end{tabular}

All predictors with a "z" prefix are standardized z-scores. Ethnicity was dummy coded, with Euro Canadians as the reference group. These results show a negative relationship between IQ and social learning with higher IQ resulting in less social learning. The regression models reported show all theoretically inspired predictors; the regression model is significant when the non-significant predictors are removed (see Supplementary Materials, available on the journal's website at www.ehbonline.org).

** $\mathrm{p}<.01$.

$* \mathrm{p}<.05$.

$* * * \quad \mathrm{p}<.001$ when data were fitted to the raw majority percentage. However, this value may inflate the tendency toward unbiased social learning because it combines individuals relying on social information with very different transmission fidelities. To address this, we scaled the majority size by the transmission fidelity and re-estimated $\alpha_{i}$. With this adjustment, the percentage of unbiased social learners dropped to $9 \%$. The remaining $91 \%$ of participants, or $85 \%$ for the unscaled calculation, showed some conformist transmission bias, with a modal strength close to 10 . These results further support the argument that fewer options underestimate the strength of the conformist transmission bias. In neither experiment did we find any evidence of anti-conformity (Morgan et al., 2014)-negative $\alpha_{i}$ values.

In Table 4, we regress the strength of the conformist transmission bias on our theoretically inspired individual predictors. Because the distribution of the $\alpha$ parameter was highly positively skewed, we took the logarithm of this value before standardizing it (see Fig. 7). For Experiment 2, we used the scaled $\alpha_{i}$ values, in part because it resulted in a better fitting model. However, no substantive differences were found using the unscaled fitted values, reported in Supplementary Materials (available on the journal's website at www.ehbonline.org).

Unlike our analysis of social learning above, the regression models in Table 4 reveal that the conformist bias is higher among those with low IQs and those with high IQs, compared to more average individuals. We found these results in both Experiments 1 and 2. We also found that the conformist bias was stronger in females and increased with age. Females had $\alpha_{i}$ values half a standard deviation higher than males, which translates to $\alpha_{i}=1.6$ higher. For age, every 5.6 years translated to an $\alpha_{i}=1.5$ increase. However, we had a limited age range with a mean age of 22. These differences were only found in Experiment 1, which is arguably more sensitive than Experiment 2, because there are often more than 2 options. 
(a)

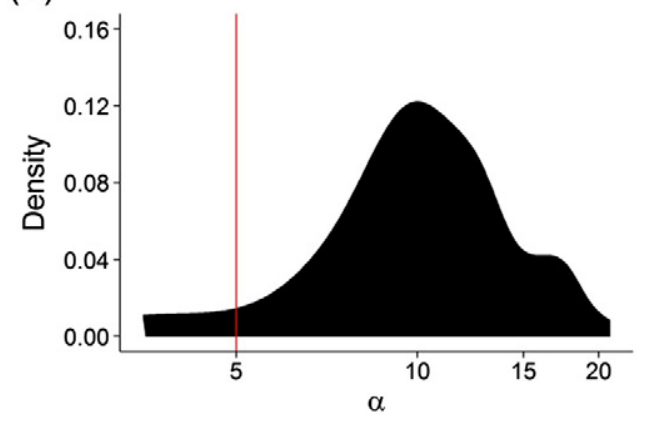

(b)

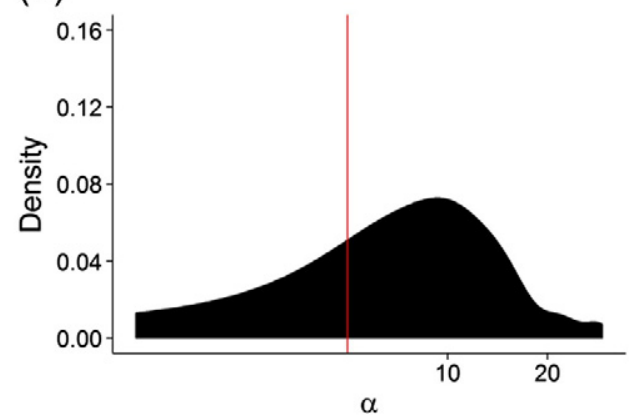

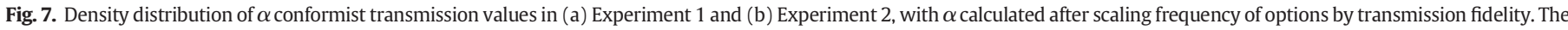

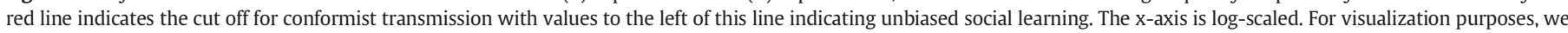
removed some outliers - see Supplementary Materials for figure including these, available on the journal's website at www.ehbonline.org.

As with social learning, other analyses revealed no effect of the other plausible predictors and no effect of increased acculturation or identification. Note that, unlike with social learning, we had no specific predictions about the effect of social status (prestige or dominance) on conformist transmission.

Given the effect of IQ on the amount of social learning and the strength of the conformist transmission bias, a reasonable question is whether these individual differences result in differences in performance and therefore payoffs. A regression analysis of performance on individual predictors revealed a consistent, but weak and nonsignificant positive effect of IQ on performance (both before and after seeing social information), suggesting that if IQ is helpful in this task, the effect is very weak (see Supplementary Materials for details, available on the journal's website at www.ehbonline.org).

\section{Discussion}

Across two experiments and an ethnically diverse sample, we tested the effect of number of options, transmission fidelity, and payoff size on the degree of social learning and the strength of the conformist bias. Our major findings can be summarized as follows:

Substantial conformist transmission. In both experiments, we found substantial reliance on conformist biased social learning, with only $3 \%$ and $9 \%$ (or $15 \%$ ) showing no conformist biases in

Table 4

OLS regression model of standardized log measures of strength of conformist transmission $(\alpha)$ regressed on our theoretical predictors as well as age and gender.

\begin{tabular}{lll}
\hline & Experiment 1 & Experiment 2 \\
\hline (Intercept) & $-0.31(-0.76,0.14)$ & $0.33(-1.64,2.29)$ \\
zIQ & $0.09(-0.16,0.33)$ & $0.09(-0.18,0.37)$ \\
zIQ & $0.26(0.11,0.41)^{* * *}$ & $0.25(0.09,0.42)^{* *}$ \\
zPrestige & $0.04(-0.18,0.26)$ & $-0.10(-0.45,0.26)$ \\
East Asian & $0.36(-0.15,0.87)$ & $-0.20(-0.77,0.36)$ \\
Other Ethnicity & $0.49(-0.11,1.10)$ & $0.07(-0.60,0.75)$ \\
zAge & $0.39(0.17,0.60)^{* * *}$ & $-0.05(-0.29,0.20)$ \\
Male & $-0.51(-0.93,0.09)^{*}$ & $0.04(-0.43,0.51)$ \\
$\mathrm{R}^{2}$ & $0.33^{* * *}$ & $0.17^{+}$ \\
$\mathrm{N}$ & 74 & 74 \\
\hline
\end{tabular}

All predictors with a "z" prefix are standardized z-scores. Ethnicity was dummy coded, with Euro Canadians as the reference group. These results suggest a consistent quadratic (U shaped) relationship between IQ and the strength of the conformist transmission bias. Both those who scored high and very low on the IQ test were more likely to have stronger conformist transmission biases than those who scored in the middle. In Experiment 1 , which is arguably more sensitive than Experiment 2 because there are often more than 2 options, conformist biases strengthen among older individuals and weaken among males.

$+\mathrm{p}<.1$.

$* \mathrm{p}<.05$.

** $\mathrm{p}<.01$.

*** $\mathrm{p}<.001$
Experiments 1 and 2, respectively. We suspect the stronger biases in Experiment 1 resulted from having multiple options at play. Two past experiments suggested no conformist bias (Coultas, 2004; Eriksson \& Coultas, 2009). These studies differed from our results in at least two critical ways making them difficult to compare. First, they did not incentivize performance or have a "right" answer. The models tested here make predictions about traits with fitness consequences. In the real world, these may be direct, e.g. eating the wrong kind of berries can get you killed, or indirect, e.g. a norm to cooperate can increase the fitness of the cooperative group. Second, conformity was operationalized with an assumption that conformist transmission requires a neutral prior. Our results show both the presence of a prior and substantial conformist transmission.

Increased social learning and stronger conformist bias as the number of options increases. Both the amount of social learning and the strength of conformist biases increased as the number of options increased, as illustrated in Figs. 3 and 4. The increase in social learning corresponds to a "copy when uncertain" strategy; uncertainty increases with number of traits. Together, these results mean that all prior experiments have merely established a lower bound on the amount of social learning and strength of conformist transmission, since all use only 2 options.

Changing inflection point with more options. The inflection point for conformist transmission behaves in a pattern consistent with the theory developed by Nakahashi et al. (2012), except that it is substantially and consistently upward biased. We suspect that this is due to a lack of any account of people's asocial priors in the model of Nakahashi et al. Future models should include asocial priors.

More reliance on social learning, but stable conformist bias across different transmission fidelities. Unexpectedly, except at very low transmission fidelities (40\% error), the strength of conformist transmission was relatively stable and flat across a wide range of transmission fidelities. Though not formally modeled, this pattern seems inconsistent with what we inferred by considering Henrich and Boyd (2002) together with Nakahashi et al. (2012). Three different factors may be relevant. First, the spatial variation typically modeled may be different from transmission errors in some fundamental way, leading us to make an inferential mistake. A proper model of transmission error is required. Another possible issue is that these results are constrained by the limited degrees of freedom in our experiment. That is, in theoretical models (and the real world) where many different types of errors can be made, conformist transmission is adaptive when transmission fidelity is low as these mistakes may result in small improvements. However, by constraining our experiment to two options, of which only one is correct, mistakes are always fatal (win-lose). New experimental designs and more data are needed to address this discrepancy. Finally, it could simply be that human psychological mechanisms are not 
designed to intuitively evaluate the format in which we provided the transmission fidelities - probabilities of accurate social information - a wealth of research suggests that people are bad at using probabilities (Tversky \& Kahneman, 1981). But, since we do observe some effects on social learning, this can't be the complete explanation.

Higher payoffs have little or no effect on learning strategies. The amount of social learning differs between no payoff and some payoff, but does not continue to increase with higher payoffs (Table 2). The strength of conformist transmission increases as the payoffs for correct answers increase. This result is not significant (Fig. 6b and c), however, Nakahashi et al. (2012) predict a very small effect, so it may be that our transmission error and payoff range were too small to detect the pattern (see Supplementary Materials Mathematica file, available on the journal's website at www. ehbonline.org). Future work might also explore the effect of different fitness landscapes on conformist biased social learning.

Higher payoffs have little or no effect on learning strategies. The amount of social learning differs between no payoff and some payoff, but does not continue to increase with higher payoffs (Table 2). The strength of conformist transmission increases as the payoffs for correct answers increase. This result is not significant (Fig. 6b and c), however, Nakahashi et al. (2012) predict a very small effect, so it may be that our transmission error and payoff range were too small to detect the pattern (see Supplementary Materials Mathematica file, available on the journal's website at www. ehbonline.org). Future work might also explore the effect of different fitness landscapes on conformist biased social learning.

Group size affects social learning with 2 options. Consistent with the theories of King and Cowlishaw (2007) and Perreault et al. (2012), we find that increased group size predicts increased social learning independent of the frequencies of options. However, we did not find this relationship for more than two options. One possibility is that with increased traits, larger groups are required for group size to have a discernible effect (our range of group sizes was 5 to 11 ).

Cognitive ability differences are associated with both social learning and the strength of the conformist bias. Extrapolating from the existing modeling work, we suspected that IQ would be negatively related to social learning and the strength of the conformist bias. This is the case for social learning, but only the case for the conformist bias in the lower range of IQs. At the upper end, higher IQs, like very low IQs, are associated with stronger conformist biases. These results together suggest that higher IQ individuals are strategically using social learning (using it less, but with a stronger conformist bias when they choose to use other information). However, IQ is only weakly related to overall performance, suggesting that, even if this is the case, these strategies are not particularly effective. Assuming our results generalize to other tasks, differences in cognitive ability may also help explain individual variation in social learning and conformist transmission in non-human species (Laland, Atton, \& Webster, 2011; Pike \& Laland, 2010).

No detectable 'cultural' differences. Neither our East Asian ethnicity variables nor our cultural identification or acculturation index pointed to any variation in social learning or conformist transmission across these populations. Nevertheless, although $53 \%$ of our sample was East Asian and $85 \%$ of them were born outside of Canada, we should take this as only preliminary evidence. It would be preferable to measure East Asians living in East Asia rather than rely on acculturation or cultural identification measures to compensate for the partial acculturation of our mostly WEIRD Canadian sample.

No detectable relationship between prestige and social learning. We predicted that individuals who view themselves as prestigious compared to others may be disinclined to copy others, because they don't see others as superior sources of information. However, we found no relationship between our measure of self-reported prestige and social learning. One reason for this might be that this general sense of prestige is psychologically very distant from the skill domain of line-length judgments, since line-length judging is not a valued skill in Vancouver. Thus, broadly prestigious individuals may not have mapped this over to the experimental task. Further research on this requires using tasks involving locally esteemed skills. No detectable relationships between other individual variables and social learning or the strength of the conformist bias. Our measures of dominance, rule-following, reflective thinking, or any of the Big 5 personality dimensions did not reliably predict social learning or the strength of the conformist bias. Thus, our results suggest that conformist biases are not a feature of personality, or other dispositional or normative tendencies like rule-following. Finally, though we were able to account for between $9 \%$ and $33 \%$ of the variance in individual's reliance on social learning and strength of conformist biases, there remains an immense amount of individual variation in these strategies that we could not explain.

Overall, our findings support the value of formal evolutionary modeling in developing and testing theories about human psychology and about social learning in particular. Broadly, they indicate that at least in this domain conformist transmission is a central component of human social learning, which varies predictably across contexts and individuals.

\section{Supplementary materials}

Supplementary data to this article can be found online at http://dx. doi.org/10.1016/j.evolhumbehav.2015.05.004.

\section{Acknowledgments}

Ethical approval for these experiments was given by the University of British Columbia Behavioral Research Ethics Board (H13-02185). All data are available from the authors upon request.

\section{References}

Aron, A., Aron, E. N., \& Smollan, D. (1992). Inclusion of other in the self scale and the structure of interpersonal closeness. Journal of Personality and Social Psychology, 63(4), 596. Bond, R., \& Smith, P. B. (1996). Culture and conformity: A meta-analysis of studies using Asch's (1952b, 1956) line judgment task. Psychological Bulletin, 119(1), 111-137, http://dx.doi.org/10.1037/0033-2909.119.1.111.

Boyd, R., \& Richerson, P. J. (1985). Culture and the evolutionary process. University of Chicago Press.

Boyd, R., \& Richerson, P. J. (1988). An evolutionary model of social learning: The effects of spatial and temporal variation. Social learning: Psychological and biological perspectives, $29-48$.

Boyd, R., \& Richerson, P. J. (1996). Why culture is common, but cultural evolution is rare. Paper presented at the Proceedings of the British Academy.

Cheng, J. T., Tracy, J. L., \& Henrich, J. (2010). Pride, personality, and the evolutionary foundations of human social status. Evolution and Human Behavior, 31(5), 334-347.

Chudek, M., Brosseau-Liard, P. E., Birch, S., \& Henrich, J. (2013). Culture-gene coevolutionary theory and children's selective social learning. In M. R. Banaji, \& S. A. Gelman (Eds.), Navigating the social world: What infants, children, and other species can teach us (pp. 181). Oxford: Oxford University Press.

Chudek, M., Muthukrishna, M., \& Henrich, J. (2015). Cultural evolution. In D. M. Buss (Ed.), The handbook of evolutionary psychology (2nd Ed.). John Wiley and Sons (in press).

Cialdini, R. B., Wosinska, W., Barrett, D. W., Butner, J., \& Gornik-Durose, M. (1999). Compliance with a request in two cultures: The differential influence of social proof and commitment/consistency on collectivists and individualists. Personality and Social Psychology Bulletin, 25(10), 1242-1253.

Coultas, J. C. (2004). When in Rome... An evolutionary perspective on conformity. Group Processes \&' Intergroup Relations, 7(4), 317-331.

Efferson, C., Lalive, R., Richerson, P. J., McElreath, R., \& Lubell, M. (2008). Conformists and mavericks: The empirics of frequency-dependent cultural transmission. Evolution and Human Behavior, 29(1), 56-64.

Eriksson, K., \& Coultas, J. (2009). Are people really conformist-biased? An empirical test and a new mathematical model. Journal of Evolutionary Psychology, 7(1), 5-21.

Eriksson, K., Enquist, M., \& Ghirlanda, S. (2007). Critical points in current theory of conformist social learning. Journal of Evolutionary Psychology, 5(1), 67-87.

Flynn, E., \& Whiten, A. (2012). Experimental "microcultures" in young children: Identifying biographic, cognitive, and social predictors of information transmission. Child Development, 83(3), 911-925, http://dx.doi.org/10.1111/j.1467-8624.2012.01747.x. 
Frederick, S. (2005). Cognitive reflection and decision making. Journal of Economic Perspectives, 25-42.

Haun, D. B. M., Rekers, Y., \& Tomasello, M. (2012). Majority-biased transmission in chimpanzees and human children, but not orangutans. Current Biology, 22(8), 727-731, http://dx.doi.org/10.1016/j.cub.2012.03.006.

Henrich, J. (2012). Too late: Models of cultural evolution and group selection have already proved useful. The False Allure of Group Selection (from http://edge.org/ conversation/the-false-allure-of-group-selection).

Henrich, J., \& Boyd, R. (1998). The evolution of conformist transmission and the emergence of between-group differences. Evolution and Human Behavior, 19(4), 215-241.

Henrich, J., \& Boyd, R. (2002). On modeling cognition and culture. Journal of Cognition and Culture, 2(2), 87-112.

Kimbrough, E. O., \& Vostroknutov, A. (2013). Norms make preferences social. Discussion papers dp13-01. Department of Economics, Simon Fraser University.

King, A. J., \& Cowlishaw, G. (2007). When to use social information: The advantage of large group size in individual decision making. Biology Letters, 3(2), 137-139.

Laland, K. N. (2004). Social learning strategies. Animal Learning E' Behavior, 32(1), 4-14.

Laland, K. N., Atton, N., \& Webster, M. M. (2011). From fish to fashion: Experimental and theoretical insights into the evolution of culture. Philosophical Transactions of the Royal Society, B: Biological Sciences, 366(1567), 958-968.

McElreath, R., Bell, A. V., Efferson, C., Lubell, M., Richerson, P. J., \& Waring, T. (2008). Beyond existence and aiming outside the laboratory: Estimating frequencydependent and pay-off-biased social learning strategies. Philosophical Transactions of the Royal Society, B: Biological Sciences, 363(1509), 3515-3528.

McElreath, R., Lubell, M., Richerson, P. J., Waring, T. M., Baum, W., Edsten, E., et al. (2005) Applying evolutionary models to the laboratory study of social learning. Evolution and Human Behavior, 26(6), 483-508.

Mesoudi, A., Chang, L., Murray, K., \& Lu, H. J. (2015). Higher frequency of social learning in China than in the West shows cultural variation in the dynamics of cultural evolution. Proceedings of the Royal Society B: Biological Sciences, 282(1798), 20142209.
Morgan, T. J. H., Laland, K. N., \& Harris, P. L. (2014). The development of adaptive conformity in young children: Effects of uncertainty and consensus. Developmental Science, http://dx.doi.org/10.1111/desc.12231.

Morgan, T., Rendell, L., Ehn, M., Hoppitt, W., \& Laland, K. (2012). The evolutionary basis of human social learning. Proceedings of the Royal Society B: Biological Sciences, 279(1729), 653-662.

Nakahashi, W., Wakano, J. Y., \& Henrich, J. (2012). Adaptive social learning strategies in temporally and spatially varying environments. Human Nature, 23(4), 386-418.

Perreault, C., Moya, C., \& Boyd, R. (2012). A Bayesian approach to the evolution of social learning. Evolution and Human Behavior, 33(5), 449-459.

Pike, T. W., \& Laland, K. N. (2010). Conformist learning in nine-spined sticklebacks' foraging decisions. Biology Letters, 6(4), 466-468.

Raven, J. C., \& Court, J. H. (1998). Raven's progressive matrices and vocabulary scales. Oxford Psychologists Press.

Rendell, L., Fogarty, L., Hoppitt, W. J., Morgan, T. J., Webster, M. M., \& Laland, K. N. (2011). Cognitive culture: Theoretical and empirical insights into social learning strategies. Trends in Cognitive Sciences, 15(2), 68-76.

Ryder, A. G., Alden, L. E., \& Paulhus, D. L. (2000). Is acculturation unidimensional or bidimensional? A head-to-head comparison in the prediction of personality, selfidentity, and adjustment. Journal of Personality and Social Psychology, 79(1), 49.

Shampanier, K., Mazar, N., \& Ariely, D. (2007). Zero as a special price: The true value of free products. Marketing Science, 26(6), 742-757.

Szabó, G., \& Tőke, C. (1998). Evolutionary prisoner's dilemma game on a square lattice. Physical Review E, 58(1), 69.

Traulsen, A., Pacheco, J. M., \& Nowak, M. A. (2007). Pairwise comparison and selection temperature in evolutionary game dynamics. Journal of Theoretical Biology, 246(3), 522-529.

Tversky, A., \& Kahneman, D. (1981). The framing of decisions and the psychology of choice. Science, 211(4481), 453-458.

Wood, L. A., Kendal, R. L., \& Flynn, E. G. (2013). Whom do children copy? Model-based biases in social learning. Developmental Review, 33(4), 341-356, http://dx.doi.org/ 10.1016/j.dr.2013.08.002. 